\title{
Desafíos actuales en la estadística oficial ${ }^{*}$
}

\author{
Pilar MARTín-GuZMÁN \\ Universidad Autónoma de Madrid, Facultad de Ciencias Económicas y Empresariales, C/ Francisco \\ Tomas y Valiente,5, 28049 Madrid, España. E-mail: pilar.guzman@uam.es
}

\begin{abstract}
RESUMEN
Las estadísticas oficiales son un instrumento para la toma de decisiones y un elemento esencial del debate político. Unas buenas estadísticas son, por tanto, imprescindibles para el buen funcionamiento de un sistema democrático.

Para que las estadísticas puedan cumplir esta función es preciso que gocen de credibilidad pública. Esto es algo que se consigue mediante el seguimiento escrupuloso y constante de unas buenas prácticas.

Pero los cambios tecnológicos, las evoluciones de la sociedad, que continuamente propone y adopta nuevos esquemas de organización y convivencia y nuevos estilos de vida, y la revolución creada por las redes sociales ponen a veces en riesgo esta credibilidad. En el Sistema Estadístico Europeo se han diseñado una serie de instrumentos dirigidos a preservar esta imagen de credibilidad. Algunos de ellos están siendo también adoptados por otras Regiones del mundo. Sin embargo, quedan aún importantes desafíos no resueltos.

Palabras clave: Credibilidad, Código de Buenas Prácticas Estadísticas, descentralización funcional, regionalización, datos masivos.
\end{abstract}

\section{Challenges in Official Statistics}

\begin{abstract}
Official statistics are an instrument for decision making and an essential tool for political debate. Good statistics are at the basis of a healthy functioning of the democratic system.

In order that official statistics can achieve their goal they need to be credible for the citizens at large. In order to win credibility a careful and permanent compliance with good practices has to be achieved.

Technological progress, the changes in a society that is permanently evolving towards new organizational frameworks and new ways of life, as well as the revolution started by the social networks can eventually put into jeopardy this credibility. The European Statistical System is implementing a number of tools aimed at preserving credibility through all these changes. Some of them are also been adopted in other Regions in the world. But there are still a number of pending challenges to face.
\end{abstract}

Keywords: Credibility, Code of Good Practices in Statistics, Functional Decentralization, Regionalization, Big Data.

Clasificación JEL: H41

\footnotetext{
* Este artículo está basado en la conferencia plenaria impartida por la autora en el XXX Congreso Internacional de Economía Aplicada, ASEPELT2016, celebrado en Valencia y que ha contado con el patrocinio de la Consellería de Educación, Investigación, Cultura y Deporte de la Generalitat Valenciana. Se agradece la financiación para la publicación de este artículo.
}

Artículo disponible en versión electrónica en la página www.revista-eea.net, ref. ə-34302 


\section{INTRODUCCIÓN}

Las estadísticas oficiales han sido desde sus orígenes un instrumento orientador para la toma de decisiones. En la medida en que sean capaces de proporcionar una representación suficientemente aproximada de la realidad económica y social, ofrecen una valiosísima ayuda en la identificación de los problemas, el diseño de las soluciones adecuadas y la evaluación a posteriori de la efectividad de las soluciones finalmente adoptadas.

Precisamente por su utilidad las estadísticas oficiales tienen una larga historia. Estadísticas básicas, como los censos de población, se han venido llevando a cabo y utilizando desde hace miles de años. Los avances teóricos surgidos a lo largo de la primera mitad del Siglo XX están permitiendo conseguir, a costes y esfuerzo razonables, una información mucho más detallada a través de las encuestas. Posteriormente, el desarrollo de la tecnología informática ha abierto a la estadística oficial perspectivas antes impensables en la obtención de información con rapidez y bajos costes a partir de los datos administrativos. La actual facilidad para disponer de grandes masas de datos en determinados sectores, juntamente con el auge de las redes sociales, podrían estar propiciando una cuarta revolución tecnológica en el campo de las estadísticas oficiales.

Pero no solamente la tecnología progresa. También la sociedad evoluciona, proponiendo y adoptando nuevos modelos de convivencia y nuevos estilos de vida. Si las estadísticas oficiales quieren mantener su utilidad como instrumentos básicos para la toma de decisiones en una determinada sociedad se van a ver continuamente enfrentadas a una serie de desafíos, que abarcan no solamente la incorporación adecuada de las nuevas tecnologías que van apareciendo, sino también su adaptación a los nuevos esquemas con los que esta sociedad se va organizando y a las nuevas formas de vida que se van implantando en ella.

Este artículo pretende exponer algunos hechos y reflexiones en torno a estos temas. Comienza comentando el cambio que se ha venido produciendo durante estos últimos años en el propio concepto de la estadística oficial, que pasa de ser un producto para uso de los gobiernos (y subsidiariamente, de los investigadores) a ser un bien de uso general por los ciudadanos. Esto da origen a una serie de desafíos, entre los cuales destaca el mantenimiento de la credibilidad de las instituciones productoras de las estadísticas. El Sistema Estadístico Europeo, consciente de este problema, ha creado una serie de instrumentos tendentes a garantizar la credibilidad de los productores de estadísticas oficiales, algunos de los cuales se están introduciendo ya en otras regiones del mundo. El último epígrafe analiza las consecuencias que ciertos cambios políticos o sociales relevantes están teniendo o van a tener presumiblemente en la disponibilidad y credibilidad de las estadísticas oficiales. 


\section{LA ESTADÍSTICA COMO BIEN PÚBLICO AL SERVICIO DEL CIUDADANO}

Tradicionalmente las estadísticas oficiales se producían para uso de los gobiernos. Y esto es lógico, puesto que en los sistemas autocráticos eran los gobiernos los encargados de la toma de decisiones.

Pero con el desarrollo de las formas de gobierno democráticas este concepto ha cambiado radicalmente. Pensemos que en nuestras sociedades actuales el círculo de usuarios de las estadísticas oficiales se ha extendido considerablemente. En un sistema democrático la oposición está igualmente interesada en ellas, pues va a utilizarlas para poder juzgar y, eventualmente, criticar, las decisiones del gobierno. También las fuerzas sociales (sindicatos, organizaciones patronales, asociaciones de consumidores y usuarios) van a emplear esta información para plantear reformas y solicitar medidas en defensa de sus intereses. Pero además, y sobre todo, en un sistema democrático el ciudadano toma periódicamente una decisión en la cual la información proporcionada por los indicadores económicos y sociales, que le van a dar la medida del éxito de las políticas adoptadas por el gobierno, va a ser un elemento determinante en su voto: El ciudadano va a decidir si apoya a ese gobierno para una nueva legislatura o si, por el contrario, se siente descontento de su actuación y, consiguientemente, confía a otro equipo diferente, a otro partido político, la gestión de su país, región o municipio.

De hecho, en un sistema democrático se supone que las decisiones son tomadas en último término por el ciudadano. Por consiguiente, es al servicio del ciudadano a lo que las estadísticas oficiales tienen que orientarse, pasando así de ser un instrumento para los gobiernos a convertirse en un bien público para uso de los ciudadanos en sentido amplio.

Esto tiene una serie de implicaciones. Los productores de estadísticas oficiales no pueden ya limitarse a cubrir la demanda de estadísticas solicitadas por los gobiernos, sino que tienen que abrirse a la sociedad de manera pro-activa, a fin de detectar cuál es la información que ésta sociedad, en continuo cambio, está necesitando, o va a necesitar presumiblemente en un futuro inmediato.

Así pues, las estadísticas oficiales juegan un papel muy importante en el desarrollo de un sistema democrático, pues ofrecen un elemento esencial de base para el debate político. Citando a Bumpstead y Aldritt podemos decir que "las estadísticas oficiales proveen información para la toma de decisiones a lo largo y ancho de toda la sociedad, y esas decisiones afectan a las vidas de todos nosotros. Son también esenciales para el entendimiento de nuestra sociedad, de nuestra economía y de la actuación del gobierno. Es, por consiguiente, axiomático, que unas buenas estadísticas oficiales son esenciales para el correcto funcionamiento de un sistema democrático". 


\section{LA CREDIBILIDAD: CONCEPTO Y FUNDAMENTOS}

Ahora bien, para que las estadísticas puedan cumplir satisfactoriamente su papel de elemento esencial en el buen funcionamiento de una democracia sana es imprescindible que los agentes sociales y la ciudadanía en general tengan confianza en ellas. Por decirlo de una forma más coloquial, es necesario que "se las crean”. Así pues, la credibilidad es para las estadísticas oficiales un atributo clave, sin el cual estas pierden toda su utilidad y su misma razón de ser.

A esto habría que añadir que la credibilidad o confianza del ciudadano es un atributo sumamente frágil, que puede perderse por errores o incidentes relativamente poco significativos, y que una vez perdida suele ser difícil de recuperar, al menos en el corto plazo.

Pero ¿en qué consiste la credibilidad? ¿Qué es lo que hace que la ciudadanía de un país confíe en sus estadísticas?

En primer lugar habría que decir que la credibilidad es fundamentalmente una percepción social. Las estadísticas se producen mediante procesos cada vez más sofisticados, y en todo caso, muy costosos. Por muy transparente que sea una Oficina de Estadística (y debe serlo al máximo nivel posible) en cuanto a la especificación de sus fuentes y metodologías, la replicación de las estadísticas rara vez es posible. La opinión pública se basa fundamentalmente en la imagen que los productores de estadísticas sean capaces de proyectar. Esta imagen se nutre, evidentemente, de elementos objetivos, pero también deja espacio a una cierta dosis de subjetividad.

Sintetizando se podría decir que los pilares de la credibilidad son a) una imagen de imparcialidad, que incluiría la independencia de la Oficina de Estadística, no solamente respecto al gobierno sino también respecto a cualquier otro posible grupo o elemento de presión y b) una imagen de calidad .

Pero al margen de la imagen que los productores de estadísticas proyecten la credibilidad puede verse seriamente afectada por factores exógenos, ajenos. Entre ellos pueden destacarse:

- La actitud general del país en cuanto se refiere a la mayor o menor confianza en sus instituciones públicas. En encuestas realizadas sobre estos temas se ha observado que los índices de credibilidad de las estadísticas oficiales de un país suelen estar bastante correlacionados con el grado de confianza que los ciudadanos de ese país tienen en sus instituciones públicas.

- La cultura estadística de la población en general y de los medios de comunicación en particular. Los medios son el enlace natural entre los productores de estadísticas y los ciudadanos, ya que estos reciben mayoritariamente a través de ellos la información estadística, en general acompañada de algún comentario. Una prensa objetiva y técnicamente bien 
preparada, que acompañe la información estadística con comentarios inteligentes, imparciales y constructivos, y que esté dispuesta a rectificar si en algún momento ha publicado interpretaciones incorrectas, es el mejor aliado de la credibilidad de las estadísticas oficiales.

\subsection{La imagen de imparcialidad}

La imagen de imparcialidad, que incluye la independencia respecto del gobierno (y de otros posibles grupos de presión) se consigue fundamentalmente mediante su ejercicio continuado a lo largo de muchos años. Hay, no obstante, una serie de elementos que contribuyen sensiblemente a su consolidación.

En primer lugar, una buena legislación estadística es una garantía pública de imparcialidad. Para que refuerce efectivamente esta imagen de imparcialidad, la legislación estadística deberá, como mínimo

- Garantizar la independencia funcional de los productores de estadísticas en cuanto a metodologías y a fechas de publicación. Hay que tener en cuenta que diferentes metodologías pueden generar resultados distintos, y esto podría dejar una puerta abierta a la arbitrariedad o incluso a la manipulación. Las metodologías a emplear en cada caso deben ser seleccionadas por los estadísticos profesionales, sin intervención o presión externa alguna, y siguiendo criterios exclusivamente técnicos. Por otra parte las fechas de la publicación de los resultados deben haber sido anunciadas en un calendario que se hace público con la suficiente antelación, y este calendario debe cumplirse rigurosamente, a fin de garantizar que la publicación de las cifras no va ser condicionada por avatares políticos.

- Establecer unas reglas objetivas para la selección y contratación del personal y para la designación de los puestos de responsabilidad en las oficinas de estadística, que garanticen la máxima imparcialidad de los estadísticos a cargo de la producción.

- Incluir alguna cláusula que garantice la confidencialidad de los datos proporcionados por los informantes, y en la que quede claramente reflejada la obligación de los productores de estadísticas oficiales de respectar rigurosamente el pacto implícito establecido con el informante, según el cual este proporciona información individualizada que la oficina de estadística facilita luego a la sociedad en forma agregada, y lo hace de manera que en ningún caso pueda ser descubierta la identidad del informante.

Pero una buena legislación no basta para mantener la imagen de imparcialidad. Esta imagen hay que cultivarla día a día mediante el seguimiento constante de unas buenas pautas de actuación, lo que se llama unas buenas prácticas. En realidad, se puede decir que las buenas prácticas cotidianas son al menos tan importantes como una buena legislación, y en todo caso, suelen ser más visibles 
para los ciudadanos. De hecho, hay en la Unión Europea países con una legislación estadística comparativamente pobre y en los que sin embargo las estadísticas oficiales gozan de un alto grado de credibilidad, cimentado en un mantenimiento permanente y escrupuloso de buenas prácticas por parte de los organismos productores.

Existe además la posibilidad de crear órganos ad-hoc que contribuyan a fomentar la credibilidad en las estadísticas. En España tenemos un buen ejemplo de esto con el Consejo Superior de Estadística. Es este un foro de encuentro entre productores y usuarios de las estadísticas oficiales en el que los diversos segmentos de usuarios, entre los cuales están los investigadores, los sindicatos, las organizaciones patronales y las asociaciones de prensa, están representados y además eligen ellos mismos a sus representantes. En este Consejo se aprueba el programa de las estadísticas que van a ser producidas, así como las metodologías que se van a emplear en cada caso. Esta transparencia ante los representantes de los usuarios y de la sociedad civil ha sido, sin duda, uno de los factores determinantes de los altos niveles de credibilidad de que actualmente goza la estadística oficial en nuestro país.

\subsection{La imagen de calidad}

El concepto de calidad de las estadísticas oficiales es algo que también está cambiando, ya que crecientemente pasa a tener en cuenta otros aspectos de la producción y a diversificar, por consiguiente los criterios de exigencia tradicionales.

Lo que tradicionalmente se entendía por calidad en las estadísticas consistía en proporcionar un retrato lo más exacto posible de una faceta de la realidad en un determinado momento. Para conseguir esto, los productores de estadísticas deben dedicar muchos recursos y esfuerzos a mantener un marco actualizado, crear diseños que minimicen el error de muestreo, reducir las tasas de no-respuesta y establecer sofisticados sistemas de depuración.

Lo que se entiende actualmente por calidad de las estadísticas es algo más amplio: su capacidad para satisfacer las necesidades de los usuarios, capacidad que, además, en las oficinas de estadística de la UE se mide periódicamente a través de la Encuesta de Satisfacción de los Usuarios, que se dirige a todos los que han sido identificados como tales a través de sus demandas o contactos mantenidos con las entidades productoras.

Evidentemente los usuarios actuales siguen solicitando calidad en su acepción tradicional, pero sus demandas no acaban ahí. Ahora quieren un buen retrato, pero además piden que este retrato esté disponible a la mayor brevedad posible. La sociedad está cambiando muy rápidamente, y los retratos proporcionados por la información estadística pierden vigencia, y, por consiguiente, utilidad para la toma de decisiones, cada día que pasa. Así pues, la rapidez en la 
producción de las estadísticas ha pasado a ser un ingrediente importante en la nueva acepción de calidad.

Esto plantea algunos problemas. Conseguir la perfección total del retrato es con frecuencia un proceso complejo y largo, y el usuario puede, con razón, preguntarse si merece la pena llegar a esos elevados niveles de precisión a costa de retrasos sustanciales en la disponibilidad final de los datos. En el dilema precisión versus rapidez los productores de estadísticas oficiales tienen que intentar situarse en el punto intermedio que mejor satisfaga las demandas de sus usuarios. Y este es, sin duda, un desafío importante.

Frente a este dilema una solución ampliamente adoptada por las oficinas de estadística de los países más avanzados es la publicación de indicadores adelantados. Se llama así a los indicadores provisionales, publicados antes de que la recogida y procesamiento de la información hayan terminado, y que tienen un carácter meramente orientativo. Posteriormente, a su debido tiempo, se publicará el indicador final que, lógicamente, tendrá casi siempre alguna ligera discrepancia con el adelantado.

Los indicadores adelantados son enormemente útiles, y muy apreciados en general por los usuarios. Sin embargo, los países cuya ciudadanía, (y muy en particular, cuya prensa) carece de una cultura estadística sólida no se atreven a publicarlos por temor a que la discrepancia entre el indicador adelantado y el final se interprete como un error, y esto ponga en riesgo su credibilidad.

Por otra parte, conseguir la perfección del retrato requiere mucho tiempo, pero también muchos recursos. Las estadísticas oficiales son un producto de alta precisión y, por tanto, de alto coste. Pero los recursos presupuestarios son siempre limitados, y aún lo están siendo más en estos últimos tiempos. Enmarcada en el espíritu de mejor servicio al usuario se ha abierto recientemente una discusión en torno a la búsqueda de un buen equilibrio calidad/coste, en la que la pregunta clave sería: ¿necesitamos siempre el retrato perfecto a costa de invertir en ello recursos que podrían, alternativamente, emplearse en ampliar el espectro de estadísticas disponibles, o incluso en otras atenciones sociales?

En las oficinas de estadística más avanzadas se habla crecientemente de adaptar los niveles de calidad de cada estadística al tipo de uso que habitualmente se hace de ella. Es lo que se conoce como fit-for-purpose. Existen estadísticas de alta sensibilidad política y social, con base a las cuales se toman decisiones fundamentales para la vida de los ciudadanos, y cuya precisión habría que cuidar al máximo. Pero hay otras que, aun aportando información relevante para el mejor conocimiento de nuestra sociedad y su evolución, no son fundamento de decisiones cruciales, y su utilización tiene un carácter más bien orientativo. En estas, niveles de precisión algo más bajos podrían suministrar, de hecho, una información equivalentemente satisfactoria para los usuarios a mucho menor coste. 
Una vez más las oficinas de estadística se ven obligadas a buscar un punto óptimo en el dilema calidad/coste. Evidentemente, decisiones de este tipo tienen que venir acompañadas de una transparencia total por parte de los productores de estadísticas, que deberán en cada caso informar de forma veraz y documentada acerca de los niveles efectivos de calidad de cada operación estadística. Las estadísticas deberán publicarse juntamente con una batería de indicadores de calidad debidamente comentados y si es posible, deberán ser clasificadas de acuerdo con su mayor o menor grado de precisión, y esta clasificación deberá también hacerse pública.

Otro aspecto que aparece crecientemente vinculado al concepto de calidad es el de la reducción de la carga al informante. Una elevada proporción de la información estadística de que disponemos actualmente depende todavía de encuestas, en las cuales la cooperación del informante es crucial. Pero en la sociedad compleja, apresurada y llena de oportunidades que nos ha tocado vivir las unidades informantes son cada vez más reacias a dedicar su tiempo a esta cooperación, y las tasas de no respuesta están aumentando significativamente.

Una forma de intentar contrarrestar esta tendencia es reducir en la medida de lo posible la carga del informante, solicitando su colaboración únicamente en los casos en que información relevante no pueda ser obtenida por otros medios, y simplificando al máximo los cuestionarios de las encuestas. De acuerdo con estos planteamientos la utilización combinada de encuestas, o de estas con registros administrativos, mediante las técnicas de matching o linking para reducir la demanda directa de información es actualmente objeto de experimentación en las oficinas de estadística más avanzadas.

\section{INSTRUMENTOS PARA LA PRESERVACIÓN DE LA CREDIBILIDAD EN EL SISTEMA ESTADÍSTICO EUROPEO}

El Sistema Estadístico Europeo, es decir, la Oficina de Estadística de la Unión Europea, EUROSTAT, juntamente con los sistemas estadísticos nacionales de los estados miembros, es consciente de la importancia de preservar al máximo su credibilidad. Con este fin ha diseñado algunos instrumentos cuyo objetivo es garantizar el seguimiento de buenas prácticas en el día a día de la producción estadística en todos los países miembros y dar la máxima publicidad a este seguimiento, contribuyendo así a potenciar la imagen de credibilidad en los usuarios y en la ciudadanía en general. Publicidad y transparencia máximas son ingredientes fundamentales de estos instrumentos, ya que su objetivo de incidir positivamente en la imagen de credibilidad difícilmente podría cumplirse si no fueran objeto de una amplia difusión entre los ciudadanos.

Los tres instrumentos fundamentales que ha creado el Sistema Estadístico Europeo con este objeto son: el Código de Buenas Prácticas de las Estadísticas Europeas, el Comité Consultivo ESGAB y las revisiones por pares. 


\subsection{El Código de Buenas Prácticas de las Estadísticas Europeas}

Los cambios políticos sobrevenidos en los años ochenta hicieron aconsejable que los principios éticos que venían rigiendo la producción de estadísticas oficiales en los países democráticos se plasmasen por escrito y sirvieran de referencia a las nuevas democracias que se iban implantando. En vista de ello, la Conferencia de Estadísticos Europeos de Naciones Unidas elaboró los Principios Fundamentales de la Estadística, que la Comisión Estadística de Naciones Unidas adoptó en 1994. Como dice el preámbulo de este documento "la necesidad de disponer de un conjunto de principios que rijan las estadísticas oficiales se hizo evidente a finales de los años ochenta, cuando los países de Europa central empezaron a cambiar de economías planificadas centralmente a democracias orientadas hacia la economía de mercado". Así surgieron los diez Principios Fundamentales, los diez mandamientos a los que todo productor de estadísticas oficiales debe atenerse.

Estos principios son, lógicamente, bastante generales, A medida que iban siendo aplicados se detectó la conveniencia de traducirlos a un documento más concreto, en el que tuviera mayor cabida la casuística que se presenta en el quehacer diario de las oficinas de estadística, y que permitiera posibles actualizaciones para afrontar los nuevos problemas que pudieran surgir. En vista de ello en el seno del Sistema Estadístico Europeo se elaboró un Código de Buenas Prácticas Estadísticas, que fue finalmente adoptado en 2005.

Su preámbulo lo describe así "El Código de Buenas Prácticas de las Estadísticas Europeas se basa en quince principios, que abarcan el entorno institucional, los procesos de elaboración de estadísticas y la producción estadística. Cada uno de estos principios va acompañado por un conjunto de indicadores de buenas prácticas que sirve de referencia para analizar la aplicación del Código. Las autoridades estadísticas, a saber, la Comisión (EUROSTAT), los institutos nacionales de estadística y otras autoridades nacionales responsables del desarrollo, la elaboración y la difusión de las estadísticas europeas, junto con los gobiernos, los ministerios y el Consejo Europeo, se comprometen a aplicar el Código".

De esta manera el Código se ha convertido en normativa de referencia para todos los productores de estadísticas oficiales en la UE. Este instrumento, que ha probado ser de gran efectividad, se está también implantando en otras regiones del mundo. Así, siguiendo en muy buena medida las directrices del Código Europeo, la Conferencia de Estadística de las Américas, una organización subsidiaria de la CEPAL (Comisión Económica para América Latina y el Caribe) de Naciones Unidas, elaboró y finalmente adoptó en el año 2011 el Código de Buenas Prácticas en Estadística para la Región. Igualmente el Foro de Estadísti- 
cos Euro-Mediterráneos ha adoptado en 2015 un Código de Buenas Prácticas Estadísticas para los Países Vecinos del Sur, ENP-South. ${ }^{1}$

\subsection{El Comité Consultivo ESGAB}

La adopción del Código de Buenas Prácticas fue, sin duda, un paso importante, al establecer unas normas claras de actuación conformes con los principios de imparcialidad y de calidad. Pero pronto se vio la conveniencia de que este paso fuese complementado con instrumentos de supervisión permanente o periódica que garantizasen a los usuarios de las estadísticas oficiales y a los ciudadanos en general el cumplimiento efectivo de los principios del Código. A este objetivo responden tanto el Comité ESGAB como las revisiones por pares.

El Comité Consultivo de la Gobernanza Estadística Europea, ESGAB (European Statistics Governance Advisory Board), fue creado conjuntamente por el Parlamento Europeo y el Consejo de Europa en el año 2008 con el objetivo de elaborar y difundir una visión independiente sobre el cumplimiento de los principios del Código de Buenas Prácticas en el Sistema Estadístico Europeo. Está constituido por siete miembros, elegidos por el Parlamento Europeo y por el Consejo de Europa entre estadísticos independientes y de reconocido prestigio de los estados de la UE. Su mandato tiene inicialmente una duración de tres años y es renovable por una sola vez.

Las misiones que fundamentalmente tiene encomendadas son:

- Elaborar anualmente un informe sobre el grado de implementación y cumplimiento de los principios del Código tanto por parte tanto de la Comisión (EUROSTAT) como de los sistemas estadísticos de los países miembros de la UE. Este informe, dirigido al Parlamento Europeo y al Consejo de Europa, se presenta oralmente ante el Parlamento Europeo en una sesión especial, en la cual los parlamentarios pueden hacer las preguntas o comentarios que estimen oportunos. Inmediatamente después se publica en la página web del Comité ${ }^{2}$, de manera que cualquier ciudadano de cualquier país del mundo tiene acceso inmediato a las opiniones y críticas contenidas en el informe.

- Revisar y dar orientaciones sobre la actualización, en su caso, del Código de Buenas Prácticas cuando los cambios sociales, la reestructuración de los sistemas estadísticos, la implantación de nuevas tecnologías o cualquier otra razón así lo aconseje.

- Aconsejar a la Comisión (EUROSTAT) sobre las medidas a adoptar para facilitar el cumplimiento de los Principios del Código y, eventualmente,

\footnotetext{
${ }^{1}$ Estos países son: Argelia, Egipto, Israel, Jordania Líbano, Marruecos, Palestina Siria, Túnez y Turquía.

${ }^{2}$ http://ec.europa.eu/eurostat/web/esgab
} 
aconsejar y apoyar igualmente a las oficinas de estadística de los países miembros. También puede asesorar a los usuarios, a petición de ellos, en particular en cuestiones relativas a la confidencialidad.

\subsection{Las revisiones por pares}

Las llamadas revisiones por pares son evaluaciones in-situ del cumplimiento de los principios del Código en EUROSTAT y en cada uno de los países miembros de la UE. Son llevadas a cabo por un equipo formado por tres estadísticos independientes, de reconocido prestigio y experiencia dentro del Sistema Estadístico Europeo, por lo que pueden ser considerados "pares” es decir, de un nivel equivalente al de los estadísticos oficiales de la máxima cualificación en el país que está siendo objeto de revisión.

Hay que tener en cuenta que, aunque las oficinas de estadística de los países miembros están generalmente dotadas por ley de una independencia funcional en el ejercicio de su actividad, son parte de la administración pública del país, y su superior jerárquico suele ser algún miembro del gobierno o. eventualmente, el Parlamento. Este es un sistema que suele funcionar bien en cuanto se refiere al control de su gestión administrativa o económica. Pero en temas tan específicos y sensibles como son los relacionados con la credibilidad y la imparcialidad resulta aconsejable una revisión por parte de un equipo independiente que además, al estar formado por estadísticos de otros estados miembros aporte el valor añadido de una mirada externa sobre el país. Este método de las revisiones por pares, que el Sistema Estadístico Europeo llevó a cabo por primera vez a lo largo del periodo 2006-2008, también está siendo adoptado por otras regiones, como América Latina y el Caribe o el grupo de los Países Vecinos del Sur, ENP-South anteriormente mencionados.

En la UE estas revisiones se repiten periódicamente cada cinco o seis años. La segunda ha sido llevada a cabo entre los años 2013 y 2015, en todos los países de la UE más los países del Espacio Económico Europeo ${ }^{3}$, y Suiza. También EUROSTAT se ha sometido a una revisión par pares, y en este caso los pares evaluadores fueron los miembros del Comité ESGAB.

Las revisiones por pares son operaciones bastante complejas, por lo que se preparan con mucha antelación. El país que va a ser revisado envía a los pares evaluadores unos dos o tres meses antes de que la evaluación vaya a tener lugar un cuestionario de autoevaluación debidamente rellenado, así como abundante documentación sobre su legislación estadística, la organización de la producción, los criterios de selección del personal, los protocolos de producción y difusión y otros aspectos de interés. Esta documentación es estudiada en detalle por el equipo evaluador que, eventualmente puede solicitar al país aclaraciones,

\footnotetext{
${ }^{3}$ Estos países son Islandia, Liechtenstein y Noruega.
} 
o documentación e información adicional si así lo considera oportuno, y que, como resultado, elaborará conjuntamente una lista de puntos que aparezcan como especialmente relevantes o sensibles en el país en cuestión.

Seguidamente tiene lugar el proceso evaluador, que consiste en una semana de entrevistas al equipo director de la oficina, a las distintas unidades productoras o difusoras, a una representación del personal de nivel intermedio, a los organismos proveedores de registros administrativos para la elaboración de estadísticas y a los distintos segmentos de usuarios: gobierno, investigadores, sindicatos, asociaciones de empresarios y, por supuesto, la prensa. En los países en que el sistema estadístico está funcionalmente descentralizado se incluyen también en esta ronda de entrevistas representantes de los organismos de la Administración Pública (Ministerios y otros) que sean productores de estadísticas oficiales.

Como resultado de todo ello se elabora un informe en el que el equipo evaluador destaca las fortalezas y las debilidades del sistema estadístico del país e incluye una serie de recomendaciones. Estos informes se publican en la web de EUROSTAT $^{4}$, es decir, son accesibles a toda persona interesada, y esta publicidad contribuye a garantizar la transparencia de los sistemas estadísticos -y, por tanto, a incrementar la credibilidad-, y supone además un estímulo a los países para mejorar la gobernanza de sus sistemas estadísticos.

Además de esto, el equipo evaluador acuerda con los representantes del país la adopción de unas acciones de mejora puntuales, concretas, que la oficina de estadística del país se compromete a llevar a cabo dentro de un horizonte temporal prefijado ${ }^{5}$. EUROSTAT se encarga de hacer un seguimiento del cumplimiento efectivo de estas acciones por parte de los países.

\section{ALGUNOS PROBLEMAS ACTUALES}

En el apartado anterior se ha puesto de manifiesto el gran esfuerzo que el Sistema Estadístico Europeo está haciendo para mantener e incrementar su credibilidad. El hecho de que varias otras Regiones del mundo estén siguiendo sus pautas es la mejor prueba de que este esfuerzo está siendo fructífero. Por otra parte, la adopción generalizada de estos instrumentos diseñados en la UE redundará, sin duda, en una consolidación de la credibilidad de las estadísticas oficiales a nivel mundial.

Pero las organizaciones políticas y sociales son dinámicas, y las estadísticas oficiales, en su intento de reflejar una realidad lo más fielmente posible, tienen

\footnotetext{
${ }^{4}$ http://ec.europa.eu/eurostat/web/quality/peer-reviews.

${ }^{5}$ En las instrucciones distribuidas por EUROSTAT a los equipos evaluadores se especifica que estas acciones de mejora deben ser SMART, acrónimo que sintetiza las siguientes características: specific, measurable, achievable, realistic y time-scaled.
} 
que adaptarse a las nuevas situaciones cuidando de no poner en riesgo su credibilidad. Los avances tecnológicos, las necesidades surgidas de la aparición de nuevos focos de interés o nuevas concepciones de la sociedad y las modificaciones en el marco político o en la estructura administrativa de los países plantean nuevos escenarios, nuevos problemas que deben ser abordados cuidadosamente. Por ejemplo:

\subsection{La descentralización de los sistemas estadísticos}

En prácticamente todos los estados miembros de la UE la producción estadística está actualmente, en mayor o menor medida, descentralizada funcionalmente. Esto quiere decir que la oficina de estadística no es el único productor de estadísticas oficiales: otros organismos, tales como los bancos centrales o los ministerios, también hacen estadísticas. Y esta descentralización tiende a crecer en la medida en que las fuentes administrativas, generalmente subproducto de registros gestionados por estos organismos, van tomando un protagonismo creciente en la producción estadística.

El Preámbulo del Código de Buenas Prácticas Estadísticas deja muy claro que el compromiso de su aplicación no se limita a los institutos nacionales de estadística, sino que se extiende también a las otras autoridades nacionales responsables del desarrollo, la elaboración y la difusión de las estadísticas europeas. Sin embargo, en algunos países de la UE la imagen de imparcialidad, de cumplimiento estricto de las normas de confidencialidad o incluso de la calidad aparece en estos organismos bastante más borrosa que en las oficinas de estadística.

En España la situación, aunque sin duda mejorable, es comparativamente mejor que en bastantes otros países miembros, ya que dentro de cada uno de estos organismos existe una unidad específicamente dedicada a la producción estadística y claramente diferenciada del resto de las unidades del correspondiente ministerio. Pero este no es siempre el caso. Hay países en los que el personal comparte la actividad estadística con otros cometidos dentro del ministerio. En ocasiones los espacios físicos no están claramente delimitados, o los ficheros de micro-datos se almacenan en sistemas informáticos compartidos por todo el organismo, con el consiguiente riesgo o, al menos, imagen de riesgo en cuanto al mantenimiento de la confidencialidad. No siempre hay una legislación que garantice la independencia funcional de estas células de producción de estadísticas, o que el personal de las mismas sea elegido con criterios estrictamente profesionales. Y no es infrecuente que, al carecer estas pequeñas unidades de oficina de prensa propia, los datos estadísticos producidos por ellas se difundan desde la oficina de prensa del ministerio y con su logo, contraviniendo así el indicador 1.6 del Principio 1 sobre la Independencia Profesional del Có- 
digo de Buenas Prácticas que dice: "Las comunicaciones estadísticas se distinguen claramente de las declaraciones políticas y se emiten al margen de éstas”.

Todo esto pone en riesgo la credibilidad del sistema estadístico en su conjunto. Con objeto de estimular el cumplimiento estricto de los principios del Código de Buenas Prácticas el Sistema Estadístico Europeo ha tomado las siguientes medidas:

- Extender el proceso de la revisión por pares a la totalidad del sistema estadístico del país, incorporando a estos otros organismos productores de estadísticas oficiales, de manera que los correspondientes informes incluyan también una descripción de sus fortalezas y debilidades y las recomendaciones subsiguientes.

- En el Reglamento (UE) 2015/759 del Parlamento Europeo y del Consejo de 29 de Abril de 2015 por el que se modifica el Reglamento (CE) 223/2009 relativo a la estadística europea el Artículo 5, apartado 1 establece que "la responsabilidad en la coordinación de los INE abarcará a todas las demás autoridades nacionales encargadas del desarrollo, la elaboración y la difusión de las estadísticas europeas...Los INE serán competentes en el plano nacional para coordinar la programación estadística y la elaboración de informes, el control de la calidad, la metodología, la transmisión de datos y la comunicación sobre las actuaciones estadísticas del Sistema Estadístico Europeo”.

Con esto se pretende, entre otras cosas, propiciar un mayor acercamiento entre las oficinas de estadística y los demás organismos productores, a fin de facilitar en ellos una más rápida difusión de la cultura del Código de Buenas Prácticas, ya bastante asentada en las oficinas centrales de estadística.

\subsection{La utilización de datos procedentes de registros administrativos}

El desarrollo de las tecnologías informáticas está permitiendo utilizar de manera creciente para la elaboración de estadísticas la información disponible en otras unidades de la administración.

El uso de registros administrativos tiene grandes ventajas respecto a las encuestas tradicionales: es incomparablemente más barato, lo que en último término ahorra costes al contribuyente, se puede procesar más rápidamente, con el consiguiente adelanto en la disponibilidad de los resultados y, por añadidura, no se molesta al informante. Pero no está exento de problemas.

En primer lugar hay un problema de acceso a estas fuentes de información. Prácticamente en todos los países de la UE hay un mandato legislativo que obliga a poner a disposición de los productores de estadísticas oficiales la información registral existente en todos los organismos de la administración. Pero no es infrecuente que existan en el país leyes de similar jerarquía que protejan 
estos registros aduciendo razones de confidencialidad o de otro tipo, lo que suele traducirse en que, finalmente, los productores de estadísticas no pueden hacer uso de esta información a nivel micro-dato.

Pero incluso cuando no existen restricciones al uso del registro administrativo, otros desafíos suelen presentarse. Por ejemplo, es fácil que haya problemas de armonización. El objetivo inicial de los datos administrativos no suele ser la elaboración de estadísticas: en general se recogen para satisfacer fines propios del organismo que los gestiona, y de ellos se extrae la información estadística como un subproducto. Si la implantación del registro no se ha llevado a cabo en colaboración con los productores de estadísticas es probable que las definiciones y clasificaciones de las variables no coincidan con las establecidas por los estándares internacionales, con lo que la elaboración, a partir de estos registros, de información estadística que sea internacionalmente comparable puede requerir un complejo proceso de adaptación o ajuste. Aún más, a medida que se producen cambios en las estructuras o hábitos sociales las definiciones y clasificaciones estadísticas internacionales se van quedando obsoletas y tienen que ser actualizadas, lo que quiere decir que los registros tienen que ser, a su vez, objeto de reajuste. Por otra parte, corresponde a los estadísticos garantizar a los ciudadanos la calidad del producto que les ofrecen, lo que les obliga a fijar unos indicadores de calidad para los registros y a comprobar su cumplimiento antes de hacer uso de estas fuentes de información.

Por último, habría que mencionar un problema que se plantea incluso en los países más avanzados en el uso de registros administrativos con fines estadísticos: el riesgo de que el organismo gestor del registro decida unilateralmente, en base a sus propios intereses u objetivos, modificar o simplemente, suprimir el registro sin la aquiescencia de los productores de estadísticas, que se ven así en la tesitura de tener que interrumpir la serie estadística correspondiente hasta tanto encuentren y pongan en explotación otras fuentes de información que puedan sustituir adecuadamente a la suprimida.

Por todo ello, el citado Reglamento (UE) 2015/759 del Parlamento Europeo y del Consejo de 29 de Abril de 2015 por el que se modifica el Reglamento (CE) 223/2009 relativo a la estadística europea ha incorporado un artículo, el 17 bis que en su apartado 1 dice:” Para reducir la carga de respuesta de los informantes los INE, las otras autoridades nacionales a las que se refiere el artículo 4 y la Comisión (EUROSTAT) tendrán derecho a acceder a todos los registros administrativos sin demora y gratuitamente, a hacer uso de ellos y a integrarlos en las estadísticas en la medida necesaria para el desarrollo, la elaboración y la difusión de las estadísticas europeas que se determinan en el Programa Estadístico Europeo de conformidad con el artículo 1". Y el apartado 2 del mismo artículo dice "Se consultará y se contará con la participación de los institutos nacionales de estadística y de la Comisión (EUROSTAT) para el diseño inicial, 
el desarrollo posterior y la supresión de registros administrativos creados y mantenidos por otros organismos, lo que facilitará el uso posterior de estos registros a efectos de la elaboración de estadísticas europeas. Los INE y la Comisión deberán participar en las actividades de estandarización relativas a registros administrativos que sean de utilidad para la generación de estadísticas europeas".

Con este artículo se pretende dar solución a los problemas anteriormente mencionados.

\subsection{Los procesos de regionalización}

A lo largo de estos últimos años se vienen dando en varios países de la UE procesos de regionalización, o de transferencia de poderes de decisión a los gobiernos regionales. España y el Reino Unido han sido ejemplo de ello, y ahora parece que Bélgica se dispone a seguir un camino parecido.

Esto ha introducido nuevos desafíos en la producción de estadísticas oficiales. Afortunadamente, estos procesos de regionalización no han afectado a la recogida de información en los censos y las encuestas, que sigue siendo en estos países responsabilidad exclusiva de las oficinas centrales de estadística, con lo que la coherencia y comparabilidad de la información quedan automáticamente garantizadas. Aquí conviene aclarar que Alemania es la excepción a esta práctica, pero ello no es en modo alguno atribuible a que Alemania haya iniciado un proceso de regionalización: se debe simplemente a que, como país, nació ya regionalizado, pues se formó por unión de estados que antes de la unificación tenían ya sus propias oficinas de estadística con sus correspondientes unidades de recogida de censos y encuestas, que por el momento se han seguido manteniendo.

Muy distinto es el caso de los registros administrativos. Al transferirse competencias a las regiones pasan a ser organismos regionales los que se encargan de gestionarlas y, por consiguiente, los que llevan los correspondientes registros. Así pues, los productores de estadísticas se ven obligados a coordinar y supervisar la calidad de registros procedentes de varias fuentes diversas, con la consiguiente dificultad añadida.

\subsection{La creciente demanda de nuevas estadísticas}

La moderna identificación del concepto de calidad como la capacidad de satisfacer las necesidades de los usuarios no deja de ser un desafío en sí misma: en la medida en que una sociedad se desarrolla económica y socialmente sus necesidades de información crecen, y tienden a ser ilimitadas. Este es ahora el caso en la UE y en los países más avanzados. La demanda de estadísticas está creciendo espectacularmente, y ello en varias direcciones y por muy diversas razones. 
Por ejemplo, los procesos de globalización están demandando de manera creciente un marco internacional, o al menos supranacional, para la confección de las estadísticas. La actividad económica de las empresas multinacionales solo puede reflejarse fielmente con un esquema multinacional. El Sistema Estadístico Europeo, consciente de este problema, está ya intentando poner en marcha el EBR (European Business Register). Por la misma razón los movimientos demográficos que se están produciendo últimamente harán pronto imprescindible un registro europeo de población. Todo esto es fuente de nuevos desafíos.

Por otra parte el proceso de atomización en la toma de decisiones que se está llevando a cabo en los países democráticos (del país a la región, de la región al municipio, del municipio al distrito) ha desatado una creciente demanda de información para áreas o entidades pequeñas, que debe también ser atendida.

Pero además aparecen continuamente nuevos enfoques, nuevas perspectivas de pensamiento, nuevos focos de interés social que también generan demanda de buenas estadísticas adicionales. Un buen ejemplo de esto es el informe Stiglitz, en el que se defiende el punto de vista de que el PIB es una medición incompleta, insuficiente, del desarrollo de las sociedades y que, por tanto, debe ser complementado con una serie de indicadores que contribuyan a medir el grado de bienestar o la calidad de vida de esta sociedad, una buena parte de los cuales tienen que ser calculados a partir de información subjetiva. De igual manera el seguimiento de los objetivos fijados por la Agenda 2030 para el Desarrollo Sostenible, publicada por Naciones Unidas en 2015 va a requerir presumiblemente la elaboración de un gran número de nuevos indicadores.

$\mathrm{Y}$ todos estos retos han de afrontarse en un momento de fuertes restricciones presupuestarias.

\subsection{La disponibilidad de datos masivos}

Uno de los problemas con que tradicionalmente han tenido que enfrentarse los productores de estadísticas oficiales es el de la competencia con el sector privado. Desde hace tiempo han venido existiendo empresas que producen estadísticas de baja calidad, pero algo más rápidas y más baratas que las oficiales.

Actualmente el desarrollo de las nuevas tecnologías y de las redes sociales lleva esta competencia a dimensiones y terrenos inimaginables hace unos años. La disponibilidad de datos masivos, los big data, permite obtener una información prácticamente inmediata, prácticamente a coste cero y sin carga adicional para los informantes. Esto abre a la producción de estadísticas perspectivas espectaculares, pero también desafíos importantes.

En primer lugar ¿qué calidad puede atribuirse a estos datos masivos? En general es una información de la cual se desconoce el marco de referencia y, por consiguiente, su grado de representatividad, y que además, está desestructurada 
de origen. En estas condiciones no parece fácil obtener a partir de ella unas estadísticas que reúnan unas mínimas garantías de calidad.

Pero además, estas masas de información suelen ser propiedad de empresas pertenecientes al sector privado, por lo que su acceso para fines estadísticos no está garantizado. La firma de convenios con las empresas propietarias de los datos masivos puede ser una solución. Sin embargo, su uso periódico para la elaboración de estadísticas no puntuales tiene algunos riesgos: al eventual incumplimiento o rescisión de los convenios se añade el riesgo de desaparición de la empresa propietaria, riesgo que no es prudente subestimar si tenemos en cuenta la volatilidad que viene caracterizando a las empresas del sector de las nuevas tecnologías.

Ahora bien, todas estas dificultades tendrán que ser salvadas de una forma o de otra. Los datos masivos están ahí, y su adecuada utilización puede generar inmensos beneficios en términos de rapidez de la información y de reducción de costes. De hecho, y aún a sabiendas de su muy dudosa calidad, estos datos seguramente van a ser utilizados a falta de información alternativa. Los productores de estadísticas oficiales no tienen más remedio que hacer frente a este complejo desafío. Si no lo hacen, la competencia con el sector privado puede endurecerse significativamente.

En resumen, la estadística oficial se enfrenta a una nueva revolución tecnológica parecida a la que se generó cuando se comenzó a utilizar las fuentes administrativas. Pero ésta promete ser bastante más compleja. Las oficinas de estadística de los países más avanzados del mundo están ya trabajando en este nuevo campo de investigación.

\section{CONCLUSIONES}

De todo ello pueden extraerse las siguientes conclusiones:

1. Las estadísticas oficiales son un elemento básico en la toma de decisiones y un instrumento esencial para el debate político. Es por esto que unas buenas estadísticas oficiales son imprescindibles para el correcto funcionamiento de un sistema democrático.

2. En un sistema democrático las decisiones son tomadas en último término por el ciudadano. Esto implica un cambio de orientación en el objetivo de las estadísticas oficiales, que pasan de ser un instrumento para los gobiernos a convertirse en un bien público para uso de los ciudadanos en general.

3. Este cambio está a su vez modificando el concepto tradicional de calidad, que ahora se centra fundamentalmente en satisfacer las necesidades de los usuarios. Estas necesidades amplían las exigencias tradicionales con otras 
nuevas, como la rapidez en la difusión de los datos, la reducción de costes y la minimización de la carga de respuesta a los informantes.

4. Para que las estadísticas oficiales puedan cumplir adecuadamente su papel de instrumentos del debate democrático necesitan gozar de credibilidad entre la población. La imagen de credibilidad es esencial y ha de mantenerse a toda costa.

5. Con el fin de mantener la credibilidad de las estadísticas oficiales el Sistema Estadístico Europeo ha puesto en marcha una serie de instrumentos que están resultando muy efectivos, por lo cual están siendo imitados en otras Regiones del mundo.

6. Estos instrumentos se revelan particularmente útiles en un momento en que las estadísticas oficiales están teniendo que enfrentarse a una serie de desafíos. Concretamente, la descentralización funcional en la producción de estadísticas oficiales, los procesos de regionalización que se están llevando a cabo en varios países de la UE y el uso creciente de las fuentes administrativas han dado lugar a una serie de problemas, a los cuales el Reglamento (UE) 2015/759 del Parlamento Europeo y del Consejo de 29 de Abril de 2015 por el que se modifica el Reglamento (CE) 223/2009 relativo a la estadística europea pretende dar solución.

7. Por otra parte, tanto los efectos de la globalización como la aparición de nuevos núcleos de interés, como pueden ser la medición del bienestar y la calidad de vida, o del desarrollo sostenible, están generando una enorme demanda en términos de nuevas estructuras estadísticas y de nuevos indicadores. Demanda que ha de ser atendida en una época de fuertes restricciones presupuestarias.

8. Los datos masivos, big data, están ya ahí, y están para ser utilizados. Pero esto presenta complejos problemas de tipo técnico (dudosa cobertura, falta de estructuración) y legal (garantía de acceso a ellos en el corto y medio plazo). Sin embargo, se perfilan como una gran oportunidad de futuro que los productores de estadísticas oficiales no pueden ignorar. Todo apunta a que una nueva revolución en las técnicas de producción de estadísticas podría estar a punto de llegar.

\section{REFERENCIAS BIBLIOGRÁFICAS}

BOHATA, M.; HAHN, M.; BAIGORRI, A. y MAíZ, G. L. (2012). "Towards a Eurostat Framework for Disseminating Innovative/Experimental Statistics". Estadística Española, nº 54, pp. 197-209. 
BUMPSTEAD, R AND ALDRITT, R , UK STATISTICAL AUTHORITY (2011). "Statistics for the people? The role of official statistics in the democratic debate". $58^{\text {Th }}$ World Congress of the International Statistical Institute, Dublin.

EUROSTAT (2011). "Código de Buenas Prácticas de las Estadísticas Europeas". http://ec.europa.eu/eurostat/documents/3859598/5922097/10425-ES-ES-PDF.

EUROPEAN STATISTICAL SYSTEM (Eurostat, EU Member States and EFTA countries) (2014) "The ESS Vision 2020". http://ec.europa.eu/eurostat/documents/ 10186/756730/ESS-vision-2020.pdf

FELLEGI, I. P. (1991). "Maintaining Public Confidence in Official Statistics". Journal of the Royal Statistical Society, Series A, vol. 154, pp. 1-6.

GARCIA, J. (2012). "La estadística oficial como bien público: retos del presente". Revista Asturiana de Economía, no 46, pp. 61-84.

GIOVANNINI, E.; OLIVEIRA MARTINS, J. y GAMBA, M. (2009). "Statistics, Knowledge and Governance". Statistika, n 6, pp. 471-490.

HOLT, T. (2008), "Official Statistics, Public Policy and Public Trust". Journal of The Royal Statistical Society, Series A, vol. 171, n² 2, pp. 323-346.

MARTÍN-GUZMÁN, P. y AGUILERA, M (2015). "Statistical Governance in the Latin American and the Caribbean Region: Achievements and Challenges". Statistical Journal of the IAOS, $n^{\circ} 31$, pp 655-659.

NORDBOTTEN, S. (2010): "The Use of Administrative Data in Official Statistics -Past, Present and Future- With Special Reference to the Nordic Countries". En Carlson, M.; Nyquist, H. y Villani, M. (eds): Official Statistics - Methodology and Applications in Honour of Daniel Thorburn. pp. 205-223.

"REGLAMENTO (UE) 2015/759 del Parlamento Europeo y del Consejo por el que se modifica el Reglamento (EC) 223/2007 relativo a la estadística europea", http://eurlex.europa.eu/legal-content/ES/TXT/PDF/?uri=CELEX;32015R0759\&from=EN

STIGLITZ, J.E.,SEN, A. and FITOUSSI, J.P. (2009). "Report by the Commission on the Measurement of Economic Performance and Social Progress". INSEE Publications, www.insee.fr/fr/publications-et-services/dossiers_web/stiglitz/doc-commission/ RAPPORT

STRUIJS,P, BRAAKSMA, B,DAAS, P.JH (2014). "Official statistics and big data". Big Data and Society, pp 1-6.

UNITED NATIONS (1994). "Fundamental Principles of Official Statistics" http://unstats.un.org/unsd/dnss/gp/fundprinciples.aspx

UNITED NATIONS (2015). "Transforming our world: The 2030 agenda for sustainable development" https://sustainabledevelopment.un.org/post2015/transformingourworld 